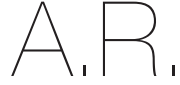
ARTIGO DE REVSÃO

Faculdade de Ciências da Nutrição e Alimentação da Universidade do Porto, Rua Dr. Roberto Frias, 4200-465 Porto, Portugal

2 CIAFEL - Centro de Investigação em Actividad Física, Saúde e Lazer da

Faculdade de Desporto da Universidade do Porto, Rua Dr. Plácido da Costa, n. ${ }^{\circ} 91$,

200-450 Porto, Portugal

Endereço para correspondência:

ernando Ribeiro Rua da Certaínha, n. ${ }^{\circ} 143$ 4630-247 Marco de Canaveses,

Portugal

fernandoribeiro1393@gmail.com

Histórico do artigo:

Recebido a 23 de maio de 201 Aceite a 10 de setembro de 2019

\section{MENTOL - O ERGOGÉNICO REFRESCANTE}

\author{
MENTHOL - THE REFRESHING ERGOGENIC
}

Fernando Ribeiro ${ }^{*}$; Vitor Hugo Teixeira ${ }^{1,2}$

\title{
RESUMO
}

O aumento da temperatura corporal é um dos principais fatores que contribui para a fadiga e diminuição da performance em provas desportivas. Por esse motivo, têm vindo a ser investigados métodos de arrefecimento corporal que visam contrariar a elevação da temperatura e potenciar a performance. Neste contexto, tem vindo a acumular-se evidência de que a ingestão de mentol pode potenciar o rendimento desportivo através de vários mecanismos não-térmicos. Entre estes, destaca-se a capacidade de ativar recetores de frio, reduzindo a perceção de calor aquando da prática de exercício intenso. Outros possíveis mecanismos incluem um aumento do volume de ar expirado, diminuição da perceção de esforço cardiopulmonar, efeitos analgésicos e efeitos estimulantes ao nível do Sistema Nervoso Central. No geral, a aplicação oral de mentol parece melhorar a performance no exercício de endurance realizado em ambiente quente e húmido, principalmente na parte final das provas. O mentol aparenta ser um composto seguro nas concentrações habitualmente usadas nos estudos em que se verificou potenciar a performance. No entanto, ao permitir prolongar o exercício para além dos limites térmicos normais, a suplementação com mentol poderá interferir no surgimento de respostas fisiológicas de proteção contra a elevação da temperatura corporal. Entretanto, é necessário testar os efeitos de concentrações maiores de mentol na performance e confirmar a sua segurança em atletas.

\section{PALAVRAS-CHAVE}

Ergogénico, Exercício de endurance, Mentol, Nutrição no desporto, Recetores de frio, Termorregulação

\section{ABSTRACT}

Increased body temperature is one of the major factors contributing to fatigue and decreased performance in sporting events. In consequence, body cooling methods have been investigated to counteract the rise in temperature and enhance performance. In this context, evidence has been accumulating that menthol intake may enhance sports performance through various non-thermal mechanisms, notably through its ability to activate cold receptors and reduce heat perception during intense exercise. Other possible mechanisms include increased expired air volume, decreased perception of cardiopulmonary effort, analgesic and CNS stimulating effects. In general, oral menthol administration seems to improve endurance exercise performance in hot and humid environments, mostly at the end of a competitive event. Menthol appears to be safe at the concentrations commonly used in studies that observed ergogenic effects with this compound. However, by allowing exercise to be prolonged beyond normal thermal limits, menthol supplementation may interfere with the arise of physiological protective responses against rising body temperature. Meanwhile, it is necessary to test the effects of higher menthol concentrations on performance and confirm its safety in athletes.

\section{KEYWORDS}

Ergogenic, Endurance exercise, Menthol, Sports nutrition, Cold receptors, Thermoregulation

\section{INTRODUÇÃO}

O organismo é muito ineficiente a transformar energia química dos alimentos em energia mecânica, sendo que $>75 \%$ é dissipada como calor (1). Se o organismo não fosse muito eficiente a termorregular, atingir-se-iam temperaturas letais em apenas 4-8 minutos (2). De facto, para que possam manter-se operacionais, os órgãos vitais da cabeça e tronco devem ser mantidos a temperaturas entre os $35-40^{\circ} \mathrm{C}$ (3). Para que o equilíbrio térmico seja mantido, é necessário que o ganho de calor seja equivalente à perda de calor, o que é conseguido através da termorregulação (3). Caso a taxa de ganho de calor exceda a taxa de dissipação para o ambiente, este irá acumular-se nos fluídos e tecidos corporais, resultando num aumento da temperatura corporal (2), o que ocorre aquando da prática de exercício físico (1). Para que este excesso de calor seja dissipado, é necessário que seja transportado para a pele, onde poderá ser transferido para o ambiente envolvente, nomeadamente através da radiação, condução, convenção e evaporação (1). Nesse contexto, o fluxo sanguíneo é redistribuído para a pele e a ativação das glândulas sudoríparas écrinas faz com 
que o suor seja secretado na superfície da pele, promovendo perda de calor por evaporação do conteúdo de água do suor (1). Se a evaporação do suor não for capaz de contrabalançar o aumento da temperatura corporal com o exercício físico, o cérebro desencadeará uma redução da intensidade ou a cessação voluntária do esforço de forma a manter a homeostase térmica $(4,5)$. Contudo, a perda de um grande volume de suor para termorregular, se não for reposto pela ingestão adequada de água, também poderá diminuir gradualmente o fluxo sanguíneo nos músculos exercitados (5). Esses fatores diminuem a capacidade de trabalho e prejudicam a performance no exercício de endurance (6-9) pelo que são úteis todas as estratégias que contribuam para os contrariar. Entre as várias estratégias existentes que poderão ajudar a prevenir o aumento da temperatura corporal, temos a aclimatização, pré-arrefecimento e arrefecimento, hiperhidratação com glicerol, reidratação durante as provas e outras $(1,10,11)$. Por exemplo, verificou-se que a ingestão de água fria e de granizados, antes ou durante o exercício, bem como outras estratégias de arrefecimento corporal, podem promover uma melhoria significativa da performance no exercício de endurance $(10,11)$. Também se sabe que alguns compostos presentes em alimentos têm a capacidade de ativar recetores de temperatura localizados (principalmente) nos nervos sensitivos do sistema nervoso periférico, suscitando dessa forma sensações de frio e calor (12). Por exemplo, a capsaícina, um composto presente na pimenta, proporciona uma sensação de calor ao ativar os recetores de potencial transitório vanilóide tipo 1 (TRPV1) (12). Ao invés, o mentol $\left(\mathrm{C}_{10} \mathrm{H}_{20} \mathrm{O}\right)$, um álcool terpeno monocíclico que ocorre naturalmente nas plantas da espécie Mentha (13), ativa os recetores de frio (12). O L-mentol é o isómero que ocorre com maior frequência na natureza e que exerce propriedades de arrefecimento não térmico mais potentes quando aplicado na pele e membranas mucosas, para além de produzir o odor característico de hortelã-pimenta (13-15). Este composto é usado, pelo menos, desde o Egipto antigo (16) e, atualmente, está presente numa grande variedade de produtos de consumo, incluindo pasta de dentes, cigarros, rebuçados, pastilhas elásticas, cosméticos, pesticidas e medicamentos para a constipação $(13,14)$. A concentração de mentol varia de 0,001\% a $2 \%$, nos produtos orais, de $0,001 \%$ a $6 \%$ nos produtos de aplicação tópica e de 0,1\% a 0,45\% nos produtos inalados (17).

\section{Efeitos e mecanismos de ação}

O mentol é o principal constituinte do óleo de hortelã-pimenta e tem a possibilidade de exercer vários efeitos fisiológicos ao se ligar a diferentes recetores. No entanto, os mecanismos fisiológicos que contribuem para os efeitos do frio e do mentol na performance do exercício de endurance ainda não estão completamente esclarecidos $(11,18,19)$. Entre os putativos efeitos atribuídos ao mentol, encontram-se os térmicos, os ventilatórios, os estimulantes e os analgésicos, por exemplo.

Efeitos térmicos: O mentol é um agonista do recetor de potencial transitório melastatina tipo 8 (TRPM8), o principal detetor de frio ambiental (20). O TRPM8 é um canal iónico transmembranar que, quando estimulado, permite o influxo de $\mathrm{Ca}^{2+} \mathrm{e} \mathrm{Na}^{+}$. Este canal possui quatro subunidades, sendo que cada uma possui seis hélices transmembranares (S1-6). Os agonistas do TRPM8 ligam-se ao S2 causando o alongamento do S4 e, consequentemente, uma modificação conformacional que conduz à abertura do canal (21). Quando em contacto com a pele ou membranas mucosas, o mentol induz sensações de frio ao estimular estes canais (TRPM8), expressos nos neurónios sensoriais A $\delta$ e C, que estão presentes maiormente na cavidade oral, língua, tecido broncopulmonar e derme superficial
$(13,21-27)$. De notar que a cavidade oral é uma das partes corporais com maior densidade de recetores periféricos (28), podendo ser uma área mais sensível aos efeitos do mentol, comparativamente à epiderme (29). Posteriormente, essa informação sensorial é transmitida ao tálamo, tronco cerebral e córtex somatossensorial $(21,30)$. Aqui, pensa-se que ocorre uma diminuição percetual do stress e desconforto térmico que, por sua vez, diminui a perceção de esforço, potenciando assim a performance (18, 31-34). Desta forma seriam minimizados os sinais inibitórios enviados para os centros de controlo motor (35), bem como a quantidade de sangue redistribuída do centro para a pele, mantendo assim a perfusão sanguínea para os músculos em atividade $(31,36)$. Também o volume de sangue ejetado e a frequência cardíaca permaneceriam constantes durante mais tempo $(31,36)$.

Efeitos ventilatórios: A administração oral de mentol causa uma sensação subjetiva de melhoria do fluxo de ar, provavelmente devido à estimulação do nervo palatino maior (37) e de termoreceptores da mucosa nasal $(37,38)$, o que poderá diminuir o esforço da respiração $(38,39)$. causar hiperventilação e, consequentemente, aumentar a capacidade de endurance (39). Ademais, vários estudos demonstraram que o mentol quando inalado é um potente broncodilatador, tendo propriedades antitússicas e atuando como descongestionante nasal $(13,40)$. Num estudo em animais verificou-se, ainda, que o vapor de mentol diminuiu a tensão de superfície entre o ar e a água do surfactante pulmonar, aumentando o nível de complacência pulmonar (41). No entanto, não parece ter a capacidade de diminuir a resistência das vias aéreas superiores $(37,38,42,43)$, o que sugere que, a esse nível, o seu efeito será apenas percetual $(42,44)$.

Efeitos estimulantes do Sistema Nervoso Central: Foi sugerido que o mentol poderá potenciar o estado de alerta através dos seus efeitos refrescantes e por promover uma maior sensação de fluxo de ar nasal que, por sua vez, poderá estimular a atividade cerebral (14). De fato, Smith e colaboradores verificaram que mascar pastilha elástica com mentol se associava a uma melhoria do estado de alerta mental (45). Sullivan et al. comprovaram que respirar uma fragrância de mentol, através de uma máscara, aumenta o nível de vigilância numa tarefa de atenção visual prolongada (46). Por sua vez, constatou-se que o aroma a mentol aumenta a velocidade e o nível de precisão em tarefas de datilografia (47). Em contraste, Zhang e colegas verificaram que chupar rebuçados de mentol não proporcionou efeitos ergogénicos/ nootrópicos durante uma simulação de combate ao fogo (48).

Efeitos analgésicos: O mentol também demonstrou inibir o canal TRPA1 (49), um mediador da dor inflamatória (50). Apesar dos efeitos analgésicos do mentol durante o exercício ainda não terem sido investigados, alguns trabalhos verificaram que, ao diminuir a perceção de esforço e a dor induzida pelo exercício, o paracetamol e outros anti-inflamatórios não esteroides têm potencial para aumentar o rendimento desportivo, uma vez que minimizam a ativação das estruturas cerebrais superiores (51). Com efeito, considera-se que a perceção da dor tem uma influência significativa no rendimento desportivo de alta intensidade (52) e é possível que o mentol também possa exercer efeitos ergogénicos através desse mecanismo $(19,53)$. Também é factível que as sensações refrescantes proporcionadas pelo mentol possam representar um estímulo "novo" que "distraia" o atleta do stress térmico e de pistas fisiológicas (34, 54), podendo ser vantajoso para o atleta envolver-se em estratégias dissociativas durante a execução de tarefas desportivas repetitivas, como o ciclismo (55).

\section{Propriedades ergogénicas}

Têm sido publicados vários trabalhos que demonstraram que o mentol tem propriedades ergogénicas, sobretudo em exercícios de endurance 
$(18,19)$. Um artigo de revisão concluiu que a prática de enxaguamento bucal intermitente com mentol, durante o exercício de endurance realizado num ambiente quente, parece beneficiar a performance neste tipo de atividade desportiva (19). Mais recentemente, foi quantificado numa meta-análise que a aplicação interna de mentol (enxaguamento bucal e/ou ingestão oral) tem um efeito pequeno ( 6\%, entre 3 e $9 \%$ ), mas significativo, na melhoria da performance de exercício de endurance, tipo contra-relógio e tempo até à exaustão, e na diminuição da perceção de sensação térmica e de esforço (18). Sugere-se que é sobretudo na parte final de provas de endurance que a aplicação interna de mentol potencia mais a performance (31, 36). A concentração de mentol usada na maioria dos estudos foi de cerca de $0,01 \%(18,19)$.

A sua utilidade também tem sido testada noutro tipo de protocolos de exercício. Assim, verificou-se que a utilização de uma fita adesiva contendo 2 gotas de óleo de menta por debaixo do nariz permitiu realizar mais flexões de tronco, correr mais rápido (400 m) e uma tendência para uma maior força de preensão da mão (56). No entanto, noutro trabalho, a inalação de aroma a mentol (57) e a ingestão de $5 \mathrm{~mL} / \mathrm{kg}$ de extrato de hortelã (58) não proporcionaram melhorias em provas de contra-relógio de 400 m. Na mesma linha, a administração oral de L-mentol (0,01\%) não parece potenciar a performance no sprint intermitente (59).

\section{Segurança}

O mentol é uma substância lipídica altamente solúvel que, após ser absorvida por via oral, através da pele ou epitélio respiratório, é transportada para o fígado através da circulação (13). Embora possa ocorrer algum metabolismo de fase 1 na pele e trato gastrointestinal, a maior parte ocorre no fígado, onde o mentol é hidroxilado por enzimas microssomais para formar sobretudo p-mentano-3,8 diol, que é posteriormente conjugado com glucuronido, que circula depois até aos rins, para ser excretado através da urina (13).

Ao diminuir a perceção de calor, o mentol poderá prolongar a realização de exercício para além dos limites térmicos normais e interferir no surgimento de respostas fisiológicas de proteção contra o aumento da temperatura corporal $(19,60)$. Para além disso, o enxaguamento bucal com fluídos contendo mentol poderá inibir a sede, sobretudo quando se utilizam fluídos frios (14), o que poderia reduzir a ingestão de líquidos, com potenciais efeitos negativos para a saúde e performance $(61,62)$.

O relatório "Eighty-sixth report of the Joint FAO/WHO Expert Committee on Food Additives", recentemente publicado, aponta para um valor máximo de Ingestão Diária Aceitável (ADI) de $4 \mathrm{mg} / \mathrm{kg}$ de peso corporal, obtido com base no nível sem efeitos adversos observados (NOAEL) de $380 \mathrm{mg} / \mathrm{kg}$ de peso corporal por dia proveniente de um estudo de 2 anos realizado em ratos (63). Tendo em conta o este valor de NOAEL e uma exposição alimentar estimada de 51,474 mg/dia, em seres humanos, a FAO/WHO concluiu que o uso do mentol como agente aromatizante não constitui uma preocupação de segurança (63), ainda que possam ser necessários mais trabalhos para definir um nível seguro para a sua ingestão (13). Entretanto, estima-se que a dose letal oral para o ser humano seja de $50-500 \mathrm{mg} / \mathrm{kg}$ de peso corporal (64).

\section{Aplicações práticas}

A ingestão de uma bebida e o enxaguamento bucal com mentol aparentam ter um efeito mais potente na performance do exercício de endurance do que a aplicação externa, através de géis ou sprays, por exemplo (19). Apesar de bastar bochechar e expetorar o mentol para se obter melhorias na performance (18), poderá ser preferível ingeri-lo juntamente com um líquido frio porque: 1) a sensação de frio na boca é, como expectável, mais intensa quando combinado com uma bebida fria $(36)$; 2$)$ um fluído frio $\left(4^{\circ} \mathrm{C}\right)$ diminui mais a tensão fisiológica, a perceção de esforço e aumenta mais a capacidade de exercício no calor (65-68); 3) os fluídos frios apesar de terem menor potencial refrescante e ergogénico que o granizado (pasta) de gelo (31, 36), são mais fáceis de ingerir durante o exercício porque não têm consistência espessa $(39,69,70)$ e têm menor probabilidade de causar desconforto gastrointestinal (71); 4) os fluídos frios são habitualmente a opção preferida (72); 5) ao estimular a via orofaríngea, o ato de engolir diminui a sensação de sede e potencia a performance (73); 6) a ingestão de fluídos frios minimiza o aumento da temperatura central (74), promove um maior arrefecimento do cérebro via transferência direta de calor com o sangue nas artérias carótidas $(31,35)$ e pode atuar em termorreceptores na zona abdominal (75).

A presença de soluções de mentol na cavidade oral, em concentrações de 0,095 a $0,105 \%$, pode estimular os nervos palatino e trigémio, o que por sua vez poderá promover o aumento da ventilação (19, $37,69,76)$ diminuir a sensação térmica $(69,76)$, a sede $(76,77)$ e melhorar o nível de conforto térmico $(31,76)$. Para formular uma bebida com uma concentração de 0,01\% de L-mentol, podemos, por exemplo, dissolver $1 \mathrm{~g}$ de cristais de mentol ((-)-menthol, Sigma Aldrich, Dorset, UK) em $100 \mathrm{ml}$ de etanol, obtendo assim uma solução com uma concentração de 1\%. Posteriormente, dilui-se a solução de etanol-mentol, adicionando $1 \mathrm{ml}$ da solução em $100 \mathrm{ml}$ de água, para obtermos uma solução final com uma concentração de 0,01\% (76). Em alternativa, pode-se esmagar e dissolver um rebuçado de Halls Extra Strong, que contém $15 \mathrm{mg}$ de mentol por rebuçado de $3,2 \mathrm{~g}(78,79)$, em cerca de $150 \mathrm{~mL}$ de água morna, para obter uma solução contendo cerca de 0,01\% de mentol. Recomenda-se que os atletas testem diferentes concentrações de L-mentol, tanto antes como durante o exercício (19), uma vez que a resposta individual à aplicação interna (oral) de mentol pode variar e irá depender de fatores fisiológicos como a espessura do estrato córneo (80), do nível de quimiossensibilidade trigeminal $(81,82)$, e da exposição habitual a agonistas do nervo trigémio $(83,84)$.

\section{ANÁLISE CRÍTICA E CONCLUSÕES}

Embora existam vários métodos para reduzir a temperatura corporal de atletas (11), a utilização da maioria deles poderá ser impraticável no decorrer de provas de endurance (85). Nesse sentido, o enxaguamento bucal ou a ingestão de fluídos contendo mentol durante o exercício poderá ser uma estratégia eficiente para diminuir a perceção de sensação térmica e potenciar a performance em atividades desportivas de endurance $(18,19)$ realizadas em ambientes quentes, sobretudo na parte final das provas $(31,36)$. No entanto, ainda são necessários mais estudos, nomeadamente que explorem e testem os efeitos fisiológicos do aumento progressivo da concentração de mentol na performance (18), bem como das diferenças entre aplicações únicas ou repetidas, quando a carga térmica é elevada (18). Além disso, dever-se-á ter em conta os potenciais efeitos deletérios da aplicação de mentol, uma vez que a diminuição da performance, aquando da exposição a temperaturas elevadas, poderá representar um mecanismo de defesa que visa proteger o organismo de lesões causadas pelo calor (86).

\section{REFERÊNCIAS BIBLIOGRÁFICAS}

1. Wendt $\mathrm{D}$, van Loon LJ, Lichtenbelt WD. Thermoregulation during exercise in the heat: strategies for maintaining health and performance. Sports medicine (Auckland, NZ). 2007;37(8):669-82. PubMed PMID: 17645370. Epub 2007/07/25. eng.

2. Hanna EG, Tait PW. Limitations to Thermoregulation and Acclimatization Challenge 
Human Adaptation to Global Warming. Int J Environ Res Public Health. 2015;12(7):8034 74. PubMed PMID: 26184272. eng.

3. Margolis HG. Heat waves and rising temperatures: human health impacts and the determinants of vulnerability. Global Climate Change and Public Health: Springer; 2014. p. 85-120.

4. Noakes TD. Maximal oxygen uptake: "classical" versus "contemporary" viewpoints a rebuttal. Medicine and science in sports and exercise. 1998 Sep;30(9):1381-98. PubMed PMID: 9741607. Epub 1998/09/19. eng.

5. Gonzalez-Alonso J. Hyperthermia impairs brain, heart and muscle function in exercising humans. Sports medicine (Auckland, NZ). 2007;37(4-5):371-3. PubMed PMID: 17465611. Epub 2007/05/01. eng.

6. Gonzalez-Alonso J, Teller C, Andersen SL, Jensen FB, Hyldig T, Nielsen B. Influence of body temperature on the development of fatigue during prolonged exercise in the heat. Journal of applied physiology (Bethesda, Md : 1985). 1999 Mar;86(3):1032-9. PubMed PMID: 10066720. Epub 1999/03/06. eng.

7. Rowell LB, Marx HJ, Bruce RA, Conn RD, Kusumi F. Reductions in cardiac output, central blood volume, and stroke volume with thermal stress in normal men during exercise. The Journal of clinical investigation. 1966;45(11):1801-16. PubMed PMID: 5926447. eng.

8. Galloway SD, Maughan RJ. Effects of ambient temperature on the capacity to perform prolonged cycle exercise in man. Medicine and science in sports and exercise. 1997 Sep;29(9):1240-9. PubMed PMID: 9309637. Epub 1997/10/06. eng.

9. Tatterson AJ, Hahn AG, Martini DT, Febbraio MA. Effects of heat stress on physiological responses and exercise performance in elite cyclists. 2000;3(2):186-93. PubMed PMID: pub.1003752221.

10. Choo HC, Nosaka K, Peiffer JJ, Ihsan M, Abbiss CR. Ergogenic effects of precooling with cold water immersion and ice ingestion: A meta-analysis. European journal of sport science. 2018 Mar;18(2):170-81. PubMed PMID: 29173092. Epub 2017/11/28. eng 11. Bongers CC, Thijssen DH, Veltmeijer MT, Hopman MT, Eijsvogels TM. Precooling and percooling (cooling during exercise) both improve performance in the heat: a metaanalytical review. British journal of sports medicine. 2015 Mar;49(6):377-84. PubMed PMID: 24747298. Epub 2014/04/22. eng

12. Vetter I, Kym PR, Szallasi A. Feeling hot, feeling cold: TRP channels-a great story unfolds. Temperature (Austin, Tex). 2015;2(2):150-1. PubMed PMID: 27227014. eng 13. Eccles R. Menthol and related cooling compounds. The Journal of pharmacy and pharmacology. 1994 Aug;46(8):618-30. PubMed PMID: 7529306. Epub 1994/08/01. eng 14. Eccles R. Role of cold receptors and menthol in thirst, the drive to breathe and arousal. Appetite. 2000 Feb;34(1):29-35. PubMed PMID: 10744889. Epub 2000/04/04. eng. 15. Eccles R, Griffiths D, Newton C, Tolley N. The effects of $D$ and $L$ isomers of menthol upon nasal sensation of airflow. The Journal of Laryngology \& Otology 1988;102(6):506-8.

16. McGovern PE, Mirzoian A, Hall GR. Ancient Egyptian herbal wines. Proceedings of the National Academy of Sciences of the United States of America. 2009;106(18):73616. PubMed PMID: 19365069. Epub 2009/04/13. eng.

17. Menthol(2014) - Workplace Environmental Exposure Level [Internet]. OARS - 2300 Montana Avenue, Suite 409, Cincinnati, OH 45211. 2014 [cited 15/04/2019]. Available from: https://www.tera.org/OARS/Menthol\%20WEEL\%2OFINAL.pdf.

18. Jeffries $O$, Waldron $M$. The effects of menthol on exercise performance and thermal sensation: A meta-analysis. Journal of science and medicine in sport. 2018 Dec 6. PubMed PMID: 30554924. Epub 2018/12/18. eng.

19. Stevens CJ, Best R. Menthol: A Fresh Ergogenic Aid for Athletic Performance. Sports medicine (Auckland, NZ). 2017 Jun;47(6):1035-42. PubMed PMID: 27858306. Epub 2016/11/20. eng.

20. Bautista DM, Siemens J, Glazer JM, Tsuruda PR, Basbaum Al, Stucky CL, et al. The menthol receptor TRPM8 is the principal detector of environmental cold. Nature. 2007 05/30/online;448:204.

21. Andersen HH, Olsen RV, Moller HG, Eskelund PW, Gazerani P, Arendt-Nielsen L. A review of topical high-concentration L-menthol as a translational model of cold allodynia and hyperalgesia. European journal of pain (London, England). 2014 Mar; 18(3):315-25. PubMed PMID: 23963768. Epub 2013/08/22. eng.
22. McKemy DD, Neuhausser WM, Julius D. Identification of a cold receptor reveals a general role for TRP channels in thermosensation. Nature. 2002 Mar 7;416(6876):528. PubMed PMID: 11882888. Epub 2002/03/08. eng.

23. Peier AM, Mogrich A, Hergarden AC, Reeve AJ, Andersson DA, Story GM, et al. A TRP channel that senses cold stimuli and menthol. Cell. 2002 Mar 8;108(5):705-15. PubMed PMID: 11893340. Epub 2002/03/15. eng.

24. Schafer K, Braun HA, Isenberg C. Effect of menthol on cold receptor activity. Analysis of receptor processes. The Journal of general physiology. 1986 Dec;88(6):75776. PubMed PMID: 3794639. PMCID: PMC2228855. Epub 1986/12/01. eng. 25. McKemy DD, Neuhausser WM, Julius D. Identification of a cold receptor reveals a general role for TRP channels in thermosensation. Nature. 2002 2002/03/01;416(6876):52-8.

26. Dhaka A, Earley TJ, Watson J, Patapoutian A. Visualizing cold spots: TRPM8expressing sensory neurons and their projections. The Journal of neuroscience : the official journal of the Society for Neuroscience. 2008 Jan 16;28(3):566-75. PubMed PMID: 18199758. Epub 2008/01/18. eng.

27. Andersen HH, Olsen RV, Møller HG, Eskelund PW, Gazerani P, Arendt-Nielsen L. A review of topical high-concentration $L$-menthol as a translational model of cold allodynia and hyperalgesia. European Journal of Pain. 2014;18(3):315-25.

28. Haggard P, de Boer L. Oral somatosensory awareness. Neuroscience and biobehavioral reviews. 2014 Nov;47:469-84. PubMed PMID: 25284337. Epub 2014/10/07. eng.

29. Green BG, Schoen KL. Thermal and nociceptive sensations from menthol and their suppression by dynamic contact. Behavioural brain research. 2007;176(2):284-91. PubMed PMID: 17092576. Epub 2006/11/07. eng.

30. Kosar E, Schwartz GJ. Effects of menthol on peripheral nerve and cortical unit responses to thermal stimulation of the oral cavity in the rat. Brain research. $1990 \mathrm{Apr}$ 16;513(2):202-11. PubMed PMID: 2350690. Epub 1990/04/16. eng.

31. Riera F, Trong TT, Sinnapah S, Hue O. Physical and perceptual cooling with beverages to increase cycle performance in a tropical climate. PLoS One. 2014;9(8):e103718. PubMed PMID: 25084009. PMCID: PMC4118924. Epub 2014/08/02. eng.

32. Flouris AD, Schlader ZJ. Human behavioral thermoregulation during exercise in the heat. Scandinavian journal of medicine \& science in sports. 2015 Jun;25 Suppl 1:52-64. PubMed PMID: 25943656. Epub 2015/05/07. eng.

33. Schlader ZJ, Simmons SE, Stannard SR, Mundel T. The independent roles of temperature and thermal perception in the control of human thermoregulatory behavior. Physiology \& behavior. 2011 May 3;103(2):217-24. PubMed PMID: 21315099. Epub 2011/02/15. eng.

34. Jeffries $\mathrm{O}$, Goldsmith M, Waldron M. L-Menthol mouth rinse or ice slurry ingestion during the latter stages of exercise in the heat provide a novel stimulus to enhance performance despite elevation in mean body temperature. European journal of applied physiology. 2018 Nov;118(11):2435-42. PubMed PMID: 30128853. PMCID: PMC6182327. Epub 2018/08/22. eng.

35. Nybo L. Hyperthermia and fatigue. Journal of applied physiology (Bethesda, Md: 1985). 2008 Mar;104(3):871-8. PubMed PMID: 17962572. Epub 2007/10/27. eng. 36. Tran Trong T, Riera F, Rinaldi K, Briki W, Hue O. Ingestion of a cold temperature/ menthol beverage increases outdoor exercise performance in a hot, humid environment. PLoS One. 2015;10(4):e0123815. PubMed PMID: 25856401. PMCID: PMC4391868. Epub 2015/04/10. eng.

37. Naito K, Komori M, Kondo Y, Takeuchi M, Iwata S. The effect of L-menthol stimulation of the major palatine nerve on subjective and objective nasal patency. Auris, nasus, larynx. 1997 Apr;24(2):159-62. PubMed PMID: 9134138. Epub 1997/04/01. eng. 38. Eccles R, Jawad MS, Morris S. The effects of oral administration of (-)-menthol on nasal resistance to airflow and nasal sensation of airflow in subjects suffering from nasal congestion associated with the common cold. The Journal of pharmacy and pharmacology. 1990 Sep;42(9):652-4. PubMed PMID: 1981905. Epub 1990/09/01. eng. 39. Mundel T, Jones DA. The effects of swilling an L(-)-menthol solution during exercise in the heat. European journal of applied physiology. 2010 May;109(1):59-65. PubMed PMID: 19727797. Epub 2009/09/04. eng.

40. Ahijevych K, Garrett BE. Menthol pharmacology and its potential impact on cigarette 
smoking behavior. Nicotine \& tobacco research : official journal of the Society for Research on Nicotine and Tobacco. 2004 Feb;6 Suppl 1:S17-28. PubMed PMID: 14982706. Epub 2004/02/26. eng.

41. Zanker KS, Tolle W, Blumel G, Probst J. Evaluation of surfactant-like effects of commonly used remedies for colds. Respiration; international review of thoracic diseases. 1980;39(3):150-7. PubMed PMID: 6893231. Epub 1980/01/01. eng. 42. Kenia P, Houghton T, Beardsmore C. Does inhaling menthol affect nasal patency or cough? Pediatric pulmonology. 2008 Jun;43(6):532-7. PubMed PMID: 18435479. Epub 2008/04/26. eng.

43. Pereira EJ, Sim L, Driver H, Parker C, Fitzpatrick M. The effect of inhaled menthol on upper airway resistance in humans: a randomized controlled crossover study. Canadian respiratory journal. 2013 Jan-Feb;20(1):e1-4. PubMed PMID: 23457678. PMCID: PMC3628651. Epub 2013/03/05. eng.

44. Nishino T, Tagaito $Y$, Sakurai $Y$. Nasal inhalation of I-menthol reduces respiratory discomfort associated with loaded breathing. American journal of respiratory and critical care medicine. 1997 Jul;156(1):309-13. PubMed PMID: 9230767. Epub 1997/07/01. eng. 45. Smith AP, Boden C. Effects of chewing menthol gum on the alertness of healthy volunteers and those with an upper respiratory tract illness. Stress and health : journal of the International Society for the Investigation of Stress. 2013 Apr;29(2):138-42. PubMed PMID: 22674677. Epub 2012/06/08. eng.

46. Sullivan TE, Warm JS, Schefft BK, Dember WN, O'Dell MW, Peterson SJ. Effects of olfactory stimulation on the vigilance performance of individuals with brain injury. Journal of clinical and experimental neuropsychology. 1998 Apr;20(2):227-36. PubMed PMID: 9777477. Epub 1998/10/20. eng.

47. Barker S, Grayhem P, Koon J, Perkins J, Whalen A, Raudenbush B. Improved performance on clerical tasks associated with administration of peppermint odor. Perceptual and motor skills. 2003 Dec;97(3 Pt 1):1007-10. PubMed PMID: 14738372. Epub 2004/01/24. eng.

48. Zhang Y, Balilionis G, Casaru C, Geary C, Schumacker RE, Neggers YH, et al. Effects of caffeine and menthol on cognition and mood during simulated firefighting in the heat. Applied ergonomics. 2014 May;45(3):510-4. PubMed PMID: 23891504 Epub 2013/07/31. eng.

49. Macpherson LJ, Hwang SW, Miyamoto T, Dubin AE, Patapoutian A, Story GM. More than cool: promiscuous relationships of menthol and other sensory compounds. Molecular and cellular neurosciences. 2006 Aug;32(4):335-43. PubMed PMID: 16829128. Epub 2006/07/11. eng.

50. Bautista DM, Pellegrino M, Tsunozaki M. TRPA1: A gatekeeper for inflammation. Annual review of physiology. 2013;75:181-200. PubMed PMID: 23020579. PMCID: PMC4041114. Epub 2012/10/02. eng

51. Holgado D, Hopker J, Sanabria D, Zabala M. Analgesics and Sport Performance: Beyond the Pain-Modulating Effects. PM \& R : the journal of injury, function, and rehabilitation. 2018 Jan;10(1):72-82. PubMed PMID: 28782695. Epub 2017/08/08. eng 52. Flood A, Waddington G, Keegan RJ, Thompson KG, Cathcart S. The effects of elevated pain inhibition on endurance exercise performance. PeerJ. 2017;5:e3028-e. PubMed PMID: 28265507. eng

53. Stevens CJ, Mauger AR, Hassmen P, Taylor L. Endurance Performance is Influenced by Perceptions of Pain and Temperature: Theory, Applications and Safety Considerations. Sports medicine (Auckland, NZ). 2018 Mar;48(3):525-37. PubMed PMID: 29270865. Epub 2017/12/23. eng.

54. St Clair Gibson A, Swart J, Tucker R. The interaction of psychological and physiological homeostatic drives and role of general control principles in the regulation of physiological systems, exercise and the fatigue process - The Integrative Governor theory. European journal of sport science. 2018 Feb;18(1):25-36. PubMed PMID: 28478704. Epub 2017/05/10. eng.

55. Bigliassi M, Karageorghis Cl, Wright MJ, Orgs G, Nowicky AV. Effects of auditory stimuli on electrical activity in the brain during cycle ergometry. Physiology \& behavior. 2017 Aug 1;177:135-47. PubMed PMID: 28442333. Epub 2017/04/27. eng. 56. Raudenbush B, Corley N, Eppich W. Enhancing athletic performance through the administration of peppermint odor. Journal of Sport and Exercise Psychology. 2001;23(2):156-60.
57. MacKenzie CM, Hedge A, editors. Is peppermint an ergogenic Aid to athletic performance? Proceedings of the Human Factors and Ergonomics Society Annual Meeting; 2005: SAGE Publications Sage CA: Los Angeles, CA.

58. Sönmez G, Çolak M, Sönmez S, Schoenfeld B. Effects of oral supplementation of mint extract on muscle pain and blood lactate. 2010;2:66. English.

59. Gibson OR, Wrightson JG, Hayes M. Intermittent sprint performance in the heat is not altered by augmenting thermal perception via L-menthol or capsaicin mouth rinses. European journal of applied physiology. 2019 Mar;119(3):653-64. PubMed PMID: 30580384. PMCID: PMC6394657. Epub 2018/12/24. eng.

60. Flood TR, Waldron M, Jeffries O. Oral L-menthol reduces thermal sensation, increases work-rate and extends time to exhaustion, in the heat at a fixed rating of perceived exertion. European journal of applied physiology. 2017 Jul;117(7):1501-12. PubMed PMID: 28508114. Epub 2017/05/17. eng.

61. Goulet ED. Effect of exercise-induced dehydration on endurance performance: evaluating the impact of exercise protocols on outcomes using a meta-analytic procedure. British journal of sports medicine. 2013 Jul;47(11):679-86. PubMed PMID: 22763119. Epub 2012/07/06. eng.

62. Liska D, Mah E, Brisbois T, Barrios PL, Baker LB, Spriet LL. Narrative Review of Hydration and Selected Health Outcomes in the General Population. Nutrients. 2019;11(1):70. PubMed PMID: 30609670. eng.

63. Organization WH. Evaluation of certain food additives: eighty-sixth report of the Joint FAOMHO Expert Committee on Food Additives. Evaluation of certain food additives: eighty-sixth report of the Joint FAO/WHO Expert Committee on Food Additives. Geneva: : World Health Organization and Food and Agriculture Organization of the United Nations; 2019.

64. Menthol - CASRN: 1490-04-6 [Internet]. National Library of Medicine HSDB Database. 2015 [cited 15/04/2019]. Available from: https://toxnet.nlm.nih.gov/cgibin/sis/search/a?dbs+hsdb:@term+@DOCNO+593.

65. Mundel T, King J, Collacott E, Jones DA. Drink temperature influences fluid intake and endurance capacity in men during exercise in a hot, dry environment. Experimental physiology. 2006 Sep;91(5):925-33. PubMed PMID: 16777932. Epub 2006/06/17. eng. 66. Lee JK, Shirreffs SM. The influence of drink temperature on thermoregulatory responses during prolonged exercise in a moderate environment. Journal of sports sciences. 2007 Jul;25(9):975-85. PubMed PMID: 17497398. Epub 2007/05/15. eng. 67. Lee JK, Maughan RJ, Shirreffs SM. The influence of serial feeding of drinks at different temperatures on thermoregulatory responses during cycling. Journal of sports sciences. 2008 Apr;26(6):583-90. PubMed PMID: 18344129. Epub 2008/03/18. eng. 68. Lee JK, Shirreffs SM, Maughan RJ. Cold drink ingestion improves exercise endurance capacity in the heat. Medicine and science in sports and exercise. 2008 Sep;40(9):1637-44. PubMed PMID: 18685527. Epub 2008/08/08. eng. 69. Stevens CJ, Thoseby B, Sculley DV, Callister R, Taylor L, Dascombe BJ. Running performance and thermal sensation in the heat are improved with menthol mouth rinse but not ice slurry ingestion. Scandinavian journal of medicine \& science in sports. 2016 Oct;26(10):1209-16. PubMed PMID: 26408395. Epub 2015/09/27. eng.

70. Stevens CJ, Bennett KJ, Sculley DV, Callister R, Taylor L, Dascombe BJ. A Comparison of Mixed-Method Cooling Interventions on Preloaded Running Performance in the Heat. Journal of strength and conditioning research. 2017 Mar;31(3):620-9. PubMed PMID: 27379961. Epub 2016/07/06. eng.

71. Stevens CJ, Thoseby B, Sculley DV, Callister R, Taylor L, Dascombe BJ. Running performance and thermal sensation in the heat are improved with menthol mouth rinse but not ice slurry ingestion. Scandinavian journal of medicine \& science in sports. 2016;26(10):1209-16.

72. Maunder E, Laursen PB, Kilding AE. Effect of ad Libitum Ice-Slurry and Cold-Fluid Ingestion on Cycling Time-Trial Performance in the Heat. International journal of sports physiology and performance. 2017 Jan;12(1):99-105. PubMed PMID: 27140841. Epub 2016/05/04. eng.

73. Adams JD, Sekiguchi Y, Suh HG, Seal AD, Sprong CA, Kirkland TW, et al. Dehydration Impairs Cycling Performance, Independently of Thirst: A Blinded Study. Medicine and science in sports and exercise. 2018 Aug;50(8):1697-703. PubMed PMID: 29509643. Epub 2018/03/07. eng. 
74. Siegel R, Mate J, Brearley MB, Watson G, Nosaka K, Laursen PB. Ice slurry ingestion increases core temperature capacity and running time in the heat. Medicine and science in sports and exercise. 2010 Apr;42(4):717-25. PubMed PMID: 19952832. Epub 2009/12/03. eng.

75. Morris NB, Bain AR, Cramer MN, Jay O. Evidence that transient changes in sudomotor output with cold and warm fluid ingestion are independently modulated by abdominal, but not oral thermoreceptors. Journal of applied physiology (Bethesda, Md: 1985). 2014 Apr 15;116(8):1088-95. PubMed PMID: 24577060. PMCID: PMC4035792. Epub 2014/03/01. eng.

76. Best R, Spears I, Hurst P, Berger N. The development of a menthol solution for use during sport and exercise. Beverages. 2018;4(2):44.

77. Eccles R, Du-Plessis L, Dommels Y, Wilkinson JE. Cold pleasure. Why we like ice drinks, ice-lollies and ice cream. Appetite. 2013 Dec;71:357-60. PubMed PMID: 24060271. Epub 2013/09/26. eng.

78. HALLS Extra Strong Menthol Flavor Drops www.gethalls.com: HAlls; [28/04/2019]. Available from: https://www.gethalls.com/relief/halls-extra-strong-menthol-flavor-drops. 79. Rebuçados Extra Strong Halls - emb. 32 gr www.continente.pt: Modelo Continente Hipermercados S.A.; 2019 [Available from: https://www.continente.pt/ stores/continente/pt-pt/public/Pages/ProductDetail.aspx?Productld=6358925(eCsf_ RetekProductCatalog_MegastoreContinenteOnline_Continente).

80. Watson H, Hems R, Rowsell D, Spring D. New compounds with the menthol cooling effect. J Soc Cosmet Chem. 1978;29(185):200.

81. Frasnelli J, Albrecht J, Bryant B, Lundstrom JN. Perception of specific trigeminal chemosensory agonists. Neuroscience. 2011 Aug 25;189:377-83. PubMed PMID: 21575683. PMCID: PMC3150232. Epub 2011/05/18. eng.

82. Michlig S, Merlini JM, Beaumont M, Ledda M, Tavenard A, Mukherjee R, et al. Effects of TRP channel agonist ingestion on metabolism and autonomic nervous system in a randomized clinical trial of healthy subjects. Scientific reports. 2016 Feb 17;6:20795. PubMed PMID: 26883089. PMCID: PMC4756362. Epub 2016/02/18. eng.

83. Cliff MA, Green BG. Sensitization and desensitization to capsaicin and menthol in the oral cavity: Interactions and individual differences. Physiology \& behavior. 1996 1996/03/01/;59(3):487-94.

84. Gillis DJ, Weston N, House JR, Tipton MJ. Influence of repeated daily menthol exposure on human temperature regulation and perception. Physiology \& behavior. 2015 Feb;139:511-8. PubMed PMID: 25484357. Epub 2014/12/09. eng.

85. Siegel R, Laursen PB. Keeping your cool: possible mechanisms for enhanced exercise performance in the heat with internal cooling methods. Sports medicine (Auckland, NZ). 2012 Feb 1;42(2):89-98. PubMed PMID: 22175533. Epub 2011/12/20. eng.

86. Nybo L. Cycling in the heat: performance perspectives and cerebral challenges. Scandinavian journal of medicine \& science in sports. 2010 Oct;20 Suppl 3:71-9. PubMed PMID: 21029193. Epub 2010/11/05. eng. 